\title{
MENINGOCOCCEMIA PRESENTING AS ACUTE SYMMETRICAL POLYARTHRITIS
}

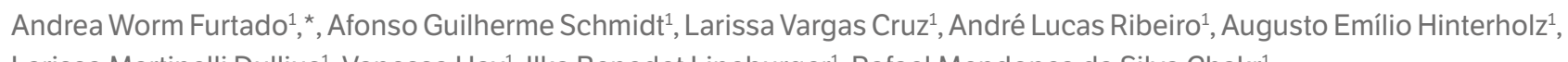
Larissa Martinelli Dullius ${ }^{1}$, Vanessa Hax¹, Ilka Benedet Lineburger ${ }^{1}$, Rafael Mendonça da Silva Chakr ${ }^{1}$

1.Hospital de Clínicas de Porto Alegre, Porto Alegre (RS), Brazil.

*Corresponding author: andreawfurtado@yahoo.com.br

\section{BACKGROUND}

The clinical spectrum of Neisseria meningitidis infection is variable, usually presenting as a meningococcemia with or without meningitis. Musculoskeletal involvement is common, manifested by myalgia and arthralgia, but the presence of arthritis is a rare complication, with a prevalence of $2-12 \%$. Septic arthritis may occur in isolation or at the time of primary meningitis or bacteremia and it is usually presented as monoarthritis or oligoarthritis, in contrast to immune complex-based arthritis, which is frequently polyarticular and a late complication of meningococcal infection. We report a case of meningococcemia with unusual initial symmetrical polyarthritis onset.

\section{CASE REPORT}

A 17-year-old male patient, previously healthy, attended the emergency department, with a four-day history of $39^{\circ} \mathrm{C}$ fever, vomiting, pleuritic chest pain and joint pain, followed by two days of acute onset of purpura. Two weeks earlier, he had presented transient flulike symptoms. Upon physical examination, there were petechiae and painful purpura on the distal extremities, pericardial friction rub and symmetrical swelling of the proximal interphalangeal joints of the hands, wrists, knees, and ankles. There was no evidence of meningeal irritation. Complementary exams showed anemia, leukocytosis, increased acute phase reactants (APR), diffuse ST-segment elevations at electrocardiogram and pericardial effusion at echocardiogram. The pericarditis findings were improved after a two-day treatment of ibuprofen $600 \mathrm{mg} 3$ times a day and colchicine $0.5 \mathrm{mg}$ twice a day, but purpura worsened, with proximal progression and cutaneous necrosis. Neisseria meningitidis was isolated from blood cultures. Other infections and autoimmune diseases were ruled out. The patient received intravenous ceftriaxone $2 \mathrm{~g}$ every $24 \mathrm{~h}$ and, after 5 days of antimicrobial regimens, showed no fever, but a moderate effusion and pain at left knee persisted. Arthrocentesis of left knee joint yielded 120,000 leukocytes $/ \mathrm{mm}^{3}$ (74\% polymorphs), with negative cultures and arthroscopic lavage was performed twice. After 14 days of IV ceftriaxone, outpatient treatment was started with intramuscular ceftriaxone $2 \mathrm{~g} /$ day for 2 weeks and oral doxycycline for 6 weeks, with a complete clinical resolution and normalization of APR.

\section{CONCLUSION}

Meningococcal disease can have a life-threatening course and mimic other infections, systemic vasculitis, drug reactions and hematologic disorders in children and young adults. High index of suspicion is needed to diagnose it in its initial stage and initiate timely antibiotics treatment. There are few case reports in medical literature that describe such an unusual presentation of symmetrical polyarthritis complication at the onset of this severe infectious disease.

\section{KEYWORDS}

Meningococcemia, Meningococcal, Polyarthritis. 